\title{
Mycophenolic acid formulations in adult renal transplantation - update on efficacy and tolerability
}

\author{
Déla Golshayan ${ }^{1,2}$ \\ M Pascual $^{2}$ \\ Bruno Vogt'
}

'Service of Nephrology and Hypertension, ${ }^{2}$ Transplantation Centre and Transplantation Immunopathology Laboratory, Department of Medicine, Centre Hospitalier Universitaire Vaudois (CHUV), Lausanne University, I0II Lausanne, Switzerland
Correspondence: Déla Golshayan Service de Néphrologie et Centre de Transplantation, BU I7, CHUV, I0I I Lausanne, Switzerland

$\mathrm{Tel}+4|2| 3|4| 939$

Fax $+4|213| 4 \mid 139$

Email dela.golshayan@chuv.ch

\begin{abstract}
The description more than 30 years ago of the role of de novo purine synthesis in $\mathrm{T}$ and $\mathrm{B}$ lymphocytes clonal proliferation opened the possibility for selective immunosuppression by targeting specific enzymatic pathways. Mycophenolic acid (MPA) blocks the key enzyme inosine monophosphate dehydrogenase and the production of guanosine nucleotides required for DNA synthesis. Two MPA formulations are currently used in clinical transplantation as part of the maintenance immunosuppressive regimen. Mycophenolate mofetil (MMF) was the first MPA agent to be approved for the prevention of acute rejection following renal transplantation, in combination with cyclosporine and steroids. Enteric-coated mycophenolate sodium (EC-MPS) is an alternative MPA formulation available in clinical transplantation. In this review, we will discuss the clinical trials that have evaluated the efficacy and safety of MPA in adult kidney transplantation for the prevention of acute rejection and their use in new combination regimens aiming at minimizing calcineurin inhibitor toxicity and chronic allograft nephropathy. We will also discuss MPA pharmacokinetics and the rationale for therapeutic drug monitoring in optimizing the balance between efficacy and safety in individual patients.
\end{abstract}

Keywords: kidney transplantation, immunosuppression, mycophenolic acid, mycophenolate mofetil, enteric-coated mycophenolate sodium, acute rejection, chronic allograft nephropathy

\section{Introduction}

The outcome of solid organ transplantation during the past two decades has been tightly linked to the development of new immunosuppressive drug therapies. ${ }^{1-4}$ The advent of calcineurin inhibitors (CNI), cyclosporine (CsA) in the 1980s and tacrolimus (TAC) in the 1990s, together with anti-T-cell induction therapies resulted in a spectacular improvement of one-year allograft survival when compared to the previous combination of azathioprine and corticosteroids. ${ }^{46}$ The primary focus of immunosuppressive protocols has always been the prevention of acute allograft rejection, a leading cause of graft loss during the first year after transplantation and a risk factor for the development of chronic allograft dysfunction and poor long-term graft outcome. However, while the rates of acute rejection episodes and short-term patient and allograft survival have steadily improved under CNI-based immunosuppressive regimens, optimal long-term allograft survival remains a problem. ${ }^{6-8}$ Indeed, despite potent anti-rejection therapies, there is still an inexorable loss of transplanted organs due to chronic allograft dysfunction, also referred to as chronic allograft nephropathy (CAN) in kidney transplantation, a process involving immunological factors and nonimmunological drug-related toxicity. ${ }^{9,10}$ Besides increased cardiovascular risk factors such as hypertension, hyperlipidemia and diabetes leading to increased patient morbidity and mortality, CNI have been associated with nephrotoxicity and vasculopathy, contributing to CAN. Thus, research efforts are now focused on finding efficacious immunosuppressive drug regimens with less graft and patient toxicity.

In this paper, we review the efficacy and safety of mycophenolic acid (MPA) formulations in human adult kidney transplantation. Based on the results of three major clinical 
trials, mycophenolate mofetil (MMF) was the first MPA agent to be approved for the prevention of acute rejection following renal transplantation, in combination with $\mathrm{CsA}$ and steroids. The results of these initial trials including long-term follow-up data of the study population and subgroup analysis will be discussed, as triple therapy of CsA-MMF-prednisone has since become the standard maintenance immunosuppression in many renal transplantation centers. We will also review trials evaluating MMF in new combination regimens aiming at minimizing CNI toxicity and CAN, as well as the use of entericcoated mycophenolate sodium (EC-MPS), an alternative MPA formulation. Finally, because of the complex pharmacokinetics of MPA resulting in inter- and intra-patient variability in drug exposure, therapeutic drug monitoring (TDM) of MPA could provide further improvement in the clinical management of individual patients and optimal immunosuppression.

\section{Cellular targets of MPA and pharmacology}

T-cells play a central role in the immune response to an allograft and most current immunosuppressive drugs target
T-cell activation and/or clonal expansion. Once T-cells have been specifically primed through their T-cell receptor (TCR) by alloantigens, the calcium-calcineurin pathway is activated, leading to the expression of survival and proinflammatory cytokines and their receptors. The engagement of the IL-2 receptor delivers growth and proliferation signals via the down-stream phophoinositide-3-kinase (PI3K) and the mammalian target-of-rapamycin (mTOR) pathways, initiating the cell cycle. Whereas CNI (CsA and TAC) inhibit the calcium-calcineurin pathway and alloantigen-dependent T-cell activation, MPA inhibits T- and B-cell proliferation (Figure 1).

The role of de novo purine synthesis in lymphocyte clonal proliferation was first highlighted by the characterization of patients with inherited immunodeficiency syndromes and the identification of key enzymatic pathways. ${ }^{11}$ MPA was originally obtained from a Penicillium fungus and shown to have anti-neoplastic, anti-viral, anti-fungal and immunosuppressive activities. ${ }^{12-14}$ MPA selectively and reversibly blocks an important step in the de novo synthesis of purine by inhibiting inosine monophosphate (IMP) dehydrogenase,

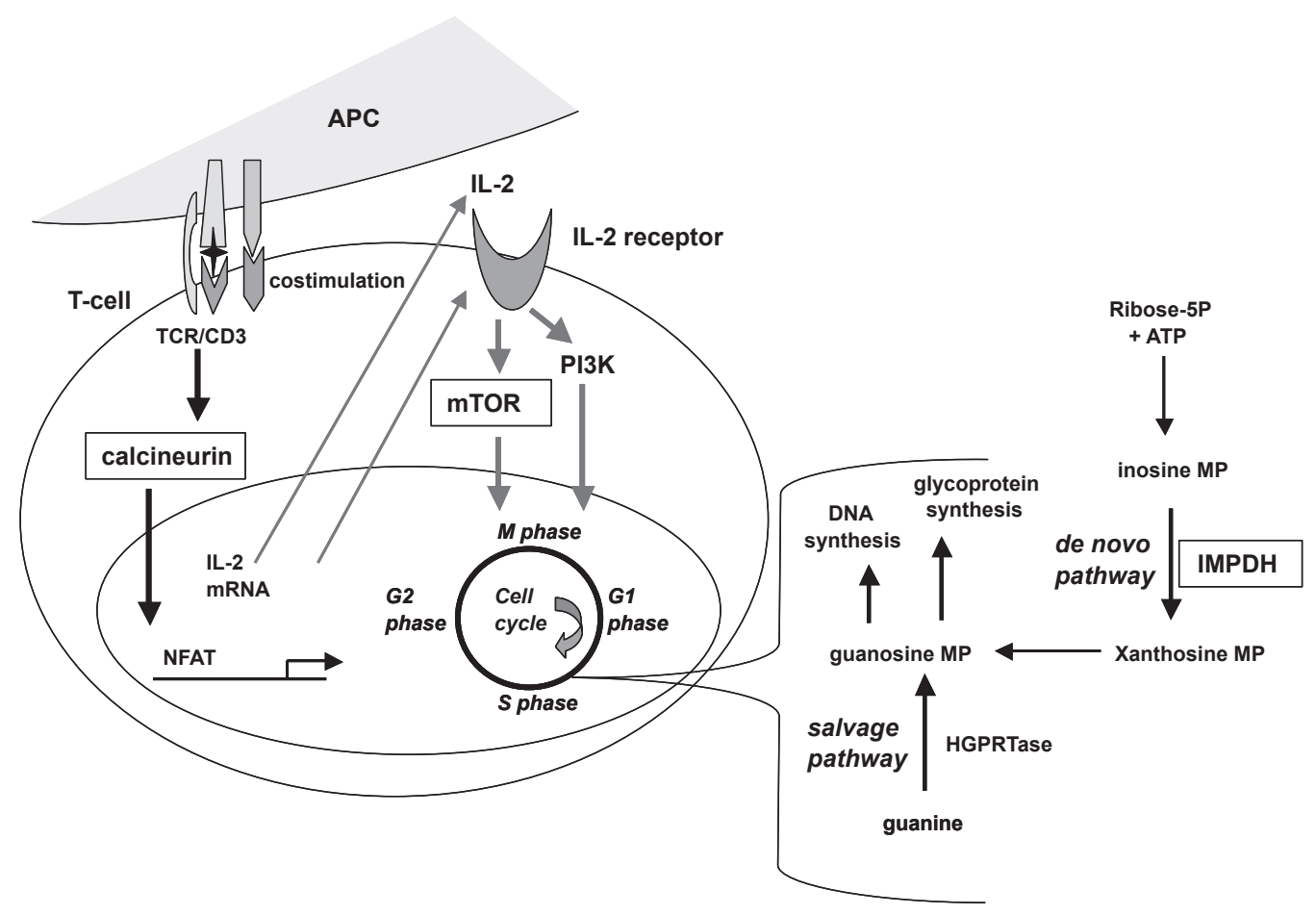

Figure I Potential targets for immunosuppressive drugs. Three distinct signals are required for the activation and differentiation of alloreactive T-cells into proliferating effector T-cells. In addition to antigen recognition, fullT-cell activation requires a costimulatory signal (signal 2 ) provided by the antigen-presenting cell (APC). The activation of signal I and 2 initiates a cascade of downstream signaling pathways and the induction of transcription factors, leading to the expression of new surface molecules such as inducible costimulatory molecules and cytokine receptors. Interleukin-2 (IL-2) and other cytokines can then deliver growth and proliferation signals (signal 3) via the phophoinositide-3-kinase (PI3K) and the mammalian target-of-rapamycin (mTOR) pathways, initiating the cell cycle.Whereas calcineurin inhibitors (cyclosporine and tacrolimus) inhibit the calcium-calcineurin pathway and alloantigen-dependent T-cell activation, anti-proliferative agents (azathioprine, mycophenolic acid [MPA], mTOR-inhibitors) inhibit lymphocyte proliferation. MPA selectively blocks an important step in the de novo synthesis of purine by inhibiting inosine monophosphate dehydrogenase (IMPDH), an essential pathway for lymphocyte division. Abbreviation: NFAT, nuclear factor of activated T cells. 
an enzyme that allows the conversion of IMP to xanthosine monophosphate, the precursor of guanosine nucleotides required for DNA synthesis (Figure 1). ${ }^{15} \mathrm{MPA}$ has no effect on the other route of purine nucleotide synthesis, the salvage pathway, where guanine obtained from the breakdown of nucleic acids is directly converted into guanosine monophosphate. Unlike neutrophils, lymphocytes depend primarily on the de novo purine synthesis pathway and may be more specifically targeted by MPA. In addition, different isoforms of IMP dehydrogenase exist, and MPA has a higher binding affinity for the type II isoform, which is upregulated in stimulated lymphocytes. Thus, MPA preferentially inhibits the division of activated lymphocytes, and, on theoretical grounds, would result in a better targeted immunosuppression with fewer side-effects that the less selective anti-metabolite azathioprine (AZA).

In vitro and in vivo studies have shown that MPA inhibits the generation of cytotoxic $\mathrm{T}$ cells and prevents antibody production by B cells, but has no direct effect on the production of cytokines. ${ }^{16}$ The synthesis of glycoproteins such as leukocyte surface adhesion molecules (selectins and integrins) is dependant on guanosine nucleotides. MPA treatment may thus also interfere with the recruitment of lymphocytes to sites of inflammation and their interaction with the endothelium of a vascularized allograft. ${ }^{17}$

\section{Mycophenolate mofetil (MMF, CellCept ${ }^{\circledR}$ )}

MMF, formerly called RS-61443, is an ester prodrug of MPA with better bioavailability (94\%). After oral administration and absorption, the ester is rapidly cleaved, releasing the active MPA component with a maximum plasma concentration at 2 hours. MPA then undergoes hepatic glucuroconjugation to form an inactive glucuronide metabolite (MPAG) which is eliminated in the urine $(90 \%)$ or excreted into the bile. In the gut, bacterial enzymes can convert this metabolite back to MPA, which is reabsorbed into circulation (enterohepatic recirculation), leading to a secondary plasma peak of MPA 6 to 12 hours after oral administration. ${ }^{18}$ The mean elimination half-life of MPA is 8 to 16 hours.

\section{Enteric-coated mycophenolate sodium (EC-MPS, Myfortic ${ }^{\circledR}$ )}

Because the use of MMF has been associated with a high incidence of gastrointestinal (GI) adverse events, a new MPA formulation was developed. EC-MPS lacks the mofetil ester and releases MPA only when the alkaline environment of the small intestine is reached. Dissolution studies with EC-MPS have shown that MPA is maximally released at
pH 6.0 to $6.8 .{ }^{19}$ Pharmacokinetic studies in renal transplant patients have demonstrated that EC-MPS $720 \mathrm{mg}$ twice a day provides an MPA exposure bioequivalent to MMF $1 \mathrm{~g}$ twice a day and has a comparable safety profile. ${ }^{19,20} \mathrm{~A}$ subsequent meta-analysis of pharmacokinetic data from three clinical trials ( $\mathrm{n}=82$ patients) confirmed the bioequivalence of EC-MPS and MMF for both MPA and metabolite exposure, and for maximum plasma MPA concentrations. ${ }^{21}$

\section{Efficacy of MMF in current immunosuppressive regimens after kidney transplantation}

Current established immunosuppressive strategies in kidney transplantation often include an induction agent (monoclonal or polyclonal anti-T-cell antibodies) based on the immunologic risk of the recipient, followed by a maintenance regimen combining a CNI and an anti-proliferative agent, with or without corticosteroids. ${ }^{2-4}$ As MMF was the first MPA formulation approved in clinical kidney transplantation, more extensive clinical data are available with this drug in comparison to EC-MPS.

\section{MMF therapy for the prevention of acute allograft rejection in cyclosporine-based regimens}

The primary objective for the addition of MMF to maintenance immunosuppression was to reduce the rate of acute rejection. Three large multicenter, prospective, randomized, double-blinded trials have evaluated MMF therapy for the prevention of acute allograft rejection in first or second renal cadaveric transplantation: the European Mycophenolate Mofetil Cooperative Study, US Renal Transplant Mycophenolate Mofetil Study Group, and the Tricontinental Mycophenolate Mofetil Renal Transplantation Study Group which was performed in Europe, Canada and Australia. ${ }^{22-24}$ Overall, these trials included 55 transplantation centers and were the largest immunosuppressive prospective randomized drug trials ever performed in transplantation. In the $U S$ and Tricontinental trials, MMF ( $\mathrm{g} /$ day and $3 \mathrm{~g}$ /day) was compared to AZA, with concomitant treatment of CsA and corticosteroids in all the groups. Anti-thymocyte globulin was given in the US trial, while patients received no induction therapy in the Tricontinental trial. In the European trial, MMF ( $2 \mathrm{~g}$ /day and $3 \mathrm{~g} /$ day) was compared to a placebo. In this trial, all the groups were also under CsA-prednisone maintenance therapy, but none received induction therapy. The primary end-point for all three trials was treatment 
failure, defined as biopsy-proven acute rejection, graft loss, patient death or withdrawal from the study during the first 6 months after transplantation. All three trials also reported drug related side-effects.

One-year efficacy data from these three trials were evaluated in a pooled analysis. ${ }^{25}$ Altogether, 1493 patients had been randomized, with 505, 490 and 498 patients in MMF $2 \mathrm{~g} /$ day, $3 \mathrm{~g} /$ day and AZA/placebo groups, respectively. The incidence of biopsy-proven acute rejection within the first year was significantly lower in transplant recipients treated with MMF (19.8\% and 16.5\% for MMF $2 \mathrm{~g} /$ day and $3 \mathrm{~g} /$ day, respectively) compared to patients receiving AZA or placebo (40.8\%). In addition, the rejection episodes were less severe in the MMF groups as evaluated by Banff scores on biopsies $(9.9 \%, 8.1 \%$ and $23.9 \%$ grade II or more severe histological scores in MMF $2 \mathrm{~g} /$ day, $3 \mathrm{~g} /$ day and AZA/placebo treatment groups, respectively) and the requirement for high-dose steroids and anti-lymphocyte antibodies. There was however no significant difference in graft function (evaluated by mean serum creatinine), nor in graft and patient survival in the first year, within the treatment groups. Of note, study withdrawal due to adverse events was more common in MMF groups $(8.7 \%, 14.7 \%$ and $5.2 \%$ in MMF $2 \mathrm{~g} / \mathrm{day}$, $3 \mathrm{~g} /$ day and AZA/placebo treatment groups, respectively). GI toxicity (mainly diarrhea, esophagitis and gastritis) was more frequent under MMF compared to AZA therapy, but in most cases improved following a decrease in MMF dose and it did not result in study discontinuation. Opportunistic infections occurred in all treatment groups but tissue-invasive CMV was more common in the MMF treatment groups. There was no report of hepatotoxicity; neutropenia and thrombocytopenia were infrequent, while anemia was similarly observed in MMF and AZA treatment groups.

Three-year follow-up data have been published for the Tricontinental and European trials. ${ }^{26,27}$ In the Tricontinental study, overall graft survival and function remained comparable in all treatment groups. However, patients who had experienced biopsy-proven rejection within 6 months after transplantation were more likely (26.1\% vs 5.7\%) to lose their transplant at 3 years compared to patients free from early acute rejection, and, graft loss due to rejection was reduced in patients receiving MMF (5.8\%, 3.0\% and 9.9\% in $\mathrm{MMF}$ $2 \mathrm{~g} /$ day, $3 \mathrm{~g} /$ day and AZA treatment groups, respectively). This illustrated the potential negative impact of early acute rejection episodes on long-term graft outcome as previously reported. ${ }^{28}$ In the European study, treatment of recipients with MMF $2 \mathrm{~g} /$ day resulted in a significant increase in graft survival at 3 years $(84.8 \%, 81.2 \%$ in MMF $2 \mathrm{~g} /$ day and
$3 \mathrm{~g} /$ day, respectively) compared to placebo (78\%). In both studies, no new significant adverse event occurred during the second and third year following transplantation. These later follow-up AZA/placebo-controlled studies have thus established the safety and efficacy of MMF treatment in renal transplantation.

Since the introduction of MMF, the original oil-based formulation of CsA has been largely replaced by the microemulsion formula of cyclosporine in immunosuppressive regimens after renal transplantation. The efficacy and tolerability of MMF in combination with CsA-microemulsion and prednisone was studied in first-time recipients of cadaveric or living-donor renal transplants. ${ }^{29}$ Treatment failure and the cumulative rate of acute rejection were significantly lower in the MMF treatment group compared with the AZA treatment group ( $p=0.007$ and $p=0.03$, respectively). Death and safety profiles were similar in both treatment groups over the12-months study period, indicating that MMF can be combined with CsA-microemulsion and prednisone for maintenance immunosuppression after renal transplantation.

\section{MMF as therapy in acute allograft rejection}

Following on these initial data, randomized trials have established the potential of MMF in the treatment of acute rejection episodes as an alternative and as an adjuvant therapy to high-dose corticosteroids ${ }^{30-32}$ in renal transplant recipients treated primarily with $\mathrm{CsA}$-prednisone maintenance immunosuppression. In combination with intravenous corticosteroids, MMF 3 g/day was significantly more effective than AZA and reduced the need for anti-lymphocyte antibodies (29.2\% vs $51.9 \%$ ). It was also shown that under MMF therapy, subsequent rejection episodes, graft loss or death were less likely compared to AZA (38.9\% vs 65.7\%) during the 3 years after transplantation. Renal function of the surviving grafts at 3 years was however similar in both treatment groups. ${ }^{32}$

Besides reducing the incidence of early acute rejection episodes, immunosuppressive protocols combining CsA and MMF have been shown to reduce late allograft rejection, thus improving long-term patient and graft survival. Data analysis of 47693 primary renal allograft recipients, reported to the United States Renal Data System (USRDS) between 1988 and 1998, has shown that long-term continuous MMF therapy was associated with a $65 \%$ decreased risk of developing late acute rejection (beyond 1 year after transplantation) as compared to AZA $(\mathrm{RR}=0.35$, CI $0.27-0.45, \mathrm{p}<0.001){ }^{33}$ In this study population the rate of acute rejection episodes in 
the AZA treatment group was $6.1 \%$ at 2 years and $9.3 \%$ at 3 years, vs $0.9 \%$ and $1.1 \%$ at 2 and 3 years after transplantation in the MMF group, respectively. ${ }^{33}$

\section{MMF therapy in combination with tacrolimus}

The initial usage of MMF in renal transplantation was in combination with CsA and steroids. With the advent of new immunosuppressive drugs, subsequent prospective randomized trials have evaluated MMF in combination regimens with these agents. Several studies have demonstrated the pharmacologic synergism between MMF and tacrolimus (TAC). ${ }^{34} \mathrm{~A}$ few large multi-center randomized trials have evaluated the risk of acute rejection in adult cadaveric kidney-transplant recipients under a combination therapy of TAC-MMF-steroids compared to TAC-steroids, TAC-AZAsteroids or CsA-MMF-steroids. ${ }^{35-39}$ TAC-MMF-steroids therapy was superior to TAC-steroids in preventing acute rejection $(27 \%$ vs $44 \%) .{ }^{35}$ In one study, TAC-AZAsteroids and TAC-MMF $1 \mathrm{~g}$ /day-steroids resulted in similar efficiency in preventing acute rejection (35,6\% vs $34.9 \%$ acute rejection episodes), while TAC-MMF 2 g/day-steroids appeared to provide greater protection $(8.9 \%)$ but without significant improvement in graft or patient survival at 1 year. Furthermore, patients in the MMF 2 g/day treatment group had their total daily dosage often reduced due to GI or hematological side-effects, so that at 6 months the mean daily dose in this group was $1.5 \mathrm{~g} /$ day. ${ }^{36}$ In another trial with 1-2- and 3-year follow-up, there was no significant difference in the incidence of acute rejection, graft and patient survival between TAC-MMF-steroids, TAC-AZA-steroids and CsA-MMF-steroids treatment groups. ${ }^{37-39}$ Median serum creatinine levels were higher in the CsA treatment group compared to the TAC-based regimens. Of note, among the subset of patients with delayed graft function, there was a significant improvement in graft survival under the TAC-MMF combination therapy $(84 \%, 70 \%$ and $61 \%$ for TAC-MMF-steroids, TAC-AZA-steroids and CsA-MMFsteroids, respectively). In summary, these studies have provided evidence that TAC-MMF-prednisone triple therapy was safe and equally effective than CsA-MMF-prednisone in preventing acute rejection in renal transplantation. Moreover the short-term results of the recent large prospective Efficacy Limiting Toxicity Elimination (ELITE) - Symphony study in de novo renal transplantation, suggest that TAC-MMFprednisone maintenance immunosuppression may even be advantageous for graft function and survival, with lower acute rejection rates. ${ }^{40}$ In this study 1645 kidney-transplant recipients were randomized in 4 treatment groups: standard-dose CsA-MMF-prednisone (CsA target trough levels $130-300 \mathrm{ng} / \mathrm{mL}$ for 3 months, then $100-200 \mathrm{ng} / \mathrm{mL}$ ), or daclizumab induction with MMF-prednisone in combination with low-dose CsA (target trough levels 50-100 ng/mL), low-dose TAC (target trough levels 3-7 ng/mL), low-dose sirolimus (SRL, target trough levels $4-8 \mathrm{ng} / \mathrm{mL}$ ). At 12 months after transplantation, a lower rate of biopsy-proven acute rejection occurred in patients receiving low-dose TAC $(12.3 \%, 25.8 \%, 24 \%, 37.2 \%$ for low-dose TAC, standarddose CsA, low-dose CsA, or low-dose SRL treatment groups, respectively) and renal function was better preserved than in the other three groups (mean calculated GFR $65.4 \mathrm{~mL} / \mathrm{min}$ vs range $56.7-59.4 \mathrm{~mL} / \mathrm{min}, \mathrm{p}<0.001)$.

\section{MMF in high-immunologic risk patients}

The reported successes of CsA-MMF-prednisone maintenance immunosuppression in adult low-immunologic risk kidneytransplant recipients has led to the evaluation of MMF safety and efficacy in high risk recipients such as in pediatric transplantation ${ }^{41}$ as well as for adult African-Americans. ${ }^{42,43}$ In a post-hoc analysis of ethnic subgroups done in the US study, ${ }^{24}$ the rate of biopsy-proven acute rejection was higher in any of the treatment groups among African-American compared to Caucasian recipients. African-Americans had more severe rejection episodes and higher serum creatinine levels at 6 months after transplantation, regardless of the treatment group, but data indicated that they had an additional benefit from the $3 \mathrm{~g}$ vs $2 \mathrm{~g} /$ day MMF dose in association with CsA and steroids $(47.5 \%, 31.8 \%, 12 \%$ incidence of acute rejection for AZA, MMF $2 \mathrm{~g}$ /day, MMF $3 \mathrm{~g} /$ day treatment groups, respectively). ${ }^{42}$

\section{MMF in CNI-sparing immunosuppressive regimens}

The addition of MMF to CNI-based maintenance immunosuppresssion has significantly reduced the rates of acute rejection in the first year after transplantation, however without a significant impact in long-term (ie, $>5$ years) graft and patient outcome. CNI are a major cause of poor graft and patient survival more that 1 year after transplantation as they can contribute to CAN and are associated with increased cardiovascular risk factors. ${ }^{7-10}$ Reducing CNI dosage or avoiding CNI could therefore be an approach to improve long-term outcome in renal transplantation. ${ }^{44}$ Thus, the introduction of potent anti-proliferative agents such as MMF in maintenance immunosuppressive regimens may allow CNI-minimization without increasing the immunological risk. 
As CNI-MMF maintenance immunosuppression was shown to prevent long-term failure of kidney grafts, independent of its effect on early and late acute rejection episodes, ${ }^{33,45,46}$ randomized multi-center prospective studies have further investigated the impact of MMF-based regimens after CNI minimization or withdrawal. The aims were either to avoid chronic nephrotoxicity in patients with stable kidney function, ${ }^{47-53}$ or to improve/maintain kidney function in the presence of CAN (defined by declining renal function and/or characteristic histological changes on protocol biopsies). ${ }^{54-61}$

In the first prospective clinical trials evaluating the safety of CsA-withdrawal strategies in renal transplantation, recipients with stable graft function on CsA-MMF-prednisone maintenance therapy over 1 year after transplantation were randomized to remain on CsA-MMF-prednisone standard therapy or MMF-prednisone treatment arm. ${ }^{47,48}$ Renal function as well as some cardiovascular risk factors such as serum cholesterol levels significantly improved after CsA withdrawal. Acute rejection rates at 6 months $(10.6 \%$ vs $2.4 \%$ ) and 5 years (16\% vs $1 \%$ ) were significantly higher in the CsA-withdrawal group versus controls, without however an impact on patient and graft survival rates at 5 years. ${ }^{48}$ To preserve renal function without increasing the risk of immunologic injury, CNI-reduction strategies (50\% dose-reduction) have been successfully reported. ${ }^{49}$

Based on the encouraging results obtained with the switch ( $>1$ year after transplantation) to low-dose CNI maintenance immunosuppression in recipients with stable renal function, multi-center prospective randomized studies investigated the efficacy and safety of de novo (ie, at the time of transplantation) low-dose CNI-MMF-prednisone and MMF-prednisone (CNI-avoidance) immunosuppressive regimens in renal transplantation. To control the immunological risk in these settings, recipients either received induction therapies with anti-T-cells antibodies ${ }^{40,50,51}$ and/or an additional anti-proliferative agent such as an mTOR-inhibitor. ${ }^{52,62-65}$ In the CAESAR study, CsA-minimization under the cover of basiliximab did not result in significant improvement of renal function and the incidence of biopsy-proven acute rejection was significantly higher in the CsA-withdrawal group (38\%) as compared to the low-dose CsA (25.4\%) or standard dose CsA (27.5\%) groups $(\mathrm{p}<0.05) .{ }^{50}$ Interestingly, in comparison, the basiliximab induction followed by low-dose TAC-MMF-prednisone immunosuppressive regimen used in the ELITE-Symphony study resulted in improved 1-year graft function with lower acute rejection rates. ${ }^{40}$

Following extensive preclinical studies, a recent multi-center randomized phase II kidney transplantation clinical trial has investigated the efficacy and safety of belatacept, a molecule that blocks T-cell activation and is not known to be nephrotoxic, as an alternative to CNI in maintenance immunosuppression. ${ }^{53}$ One-year results showed similar acute rejection rates in both arms (19\% vs $18 \%$ in belatacept-MMF-prednisone and CsA-MMF-prednisone groups, respectively), while in the belatacept treatment group renal function was significantly improved with a lower prevalence of CAN on protocol biopsies.

CNI dose-reduction was also attempted under the cover of MMF-prednisone in recipients with declining renal function and CAN confirmed on biopsies. ${ }^{54-61}$ In general, in these transplant recipients, serum creatinine levels stabilized or decreased after CNI-minimization and the switch from AZA-prednisone to MMF-prednisone maintenance immunosuppression, without an increase in the incidence of acute rejection. Thus, these observational and randomized clinical trials suggest that it could be a safe and effective therapeutic option in selected renal transplant recipients with worsening renal function. Furthermore, the reduction or complete withdrawal of CNI could also improve metabolic parameters and blood pressure control and decrease patient's cardiovascular morbidity. ${ }^{61}$

\section{Safety and efficacy of EC-MPS versus MMF \\ Side-effects associated with MMF}

The majority of data concerning the safety and tolerability of MMF in transplant recipients were obtained from the three earliest prospective randomized double-blinded clinical trials in renal transplantation, evaluating MMF at $2 \mathrm{~g}$ /day and 3 g/day. ${ }^{22-24}$ Long-term trials of patients with psoriasis treated with MMF are also available. ${ }^{66}$ Overall, MMF was well tolerated with most of the symptoms being dose-dependant as the reported side-effects generally resolved with the reduction of the dose. In these studies, GI toxicity (mainly diarrhea, nausea, esophagitis and gastritis) and tissueinvasive CMV were more common with MMF therapy, while myelosuppression (mainly leucopenia and anemia, only rarely neutropenia) and other opportunistic infections also occurred in AZA treatment groups. GI side-effects can occur in up to $45 \%$ of patients treated with MMF and are in part due to systemic MPA exposure and its antiproliferative effect on enterocytes. ${ }^{22-24,67}$ Dose reductions or temporarily discontinuation of MMF may be sufficient to relieve the symptoms but can result in sub-therapeutic levels.

EC-MPS is an alternative formulation of MPA, designed to reduce GI toxicity. In a few multi-center prospective open-label studies, MMF-treated kidney-transplant patients 
experiencing GI complaints were successfully converted to equimolar doses of EC-MPS. ${ }^{68,69}$ These studies have shown that following the switch to EC-MPS, GI-related symptoms and health-related quality of life, as assessed by self-administered patient questionnaires, improved significantly ${ }^{68}$ and this was a sustained effect over time after conversion to EC-MPS. ${ }^{69}$

\section{Bioequivalence, safety and tolerability of EC-MPS in renal transplantation}

Randomized clinical trials in renal transplantation have evaluated MPA drug exposure and the bioequivalence of EC-MPS compared to MMF, as well as the therapeutic efficacy and safety of EC-MPS in renal transplantation, either de novo after transplantation as part of CNI-based maintenance immunosuppression or after a switch from MMF. ${ }^{70-72}$ The ERL B301 Study group, an international, randomized, double-blind trial, has assessed the therapeutic equivalence of EC-MPS (720 mg bid) and MMF (1000 mg bid), with concomitant CsA microemulsion and corticosteroids, in 423 de novo kidney-transplant patients. ${ }^{70,71}$ At 12 months, the incidence of biopsy proven acute rejection, graft loss or death was $26.3 \%$ and $28.1 \%$ (biopsy proven acute rejection alone $22.5 \%$ and $24.3 \%$ ) for EC-MPS and MMF, respectively. The safety profile and interestingly the incidence of GI adverse events were similar for both groups. ${ }^{70}$ In the extension study, after 1 year of treatment the patients first randomized in the MMF group were converted to EC-MPS, and both groups were followed for 2 more years. ${ }^{71}$ The overall incidence of adverse events, acute rejection and graft loss during the extension phase was comparable to that seen in the core study, and in the MMF arm of other similar controlled randomized studies. This study established the long-term efficacy and tolerability of EC-MPS in association with CsA and steroids, as well as the safety of converting renal transplant patients from MMF to EC-MPS.

The myfortic Prospective Multicentre Study ( $m y$ PROMS) was an international, prospective, open-label, clinical trial designed to assess the efficacy and safety of EC-MPS in combination with $\mathrm{CsA}$ microemulsion in a large population of kidney-transplant recipients. ${ }^{73-75}$ The study had a predefined core protocol with 14 sub-studies addressing different aspects related to EC-MPS treatment. All participants were treated with EC-MPS and CsA microemulsion (C2 level monitoring), with or without corticosteroids, and induction therapy was given as per center practice. The primary efficacy evaluation was the rate of treatment failure, defined as biopsy-proven acute rejection, graft loss or death of the recipient during the first 6 and 12 months after transplantation. Safety and tolerability analyses were also performed for all patients, including reported adverse-events, hematology and chemistry profiles, infections and malignancies. Data were pooled from multiple centers for planned analysis of sub-protocols.

In a 6-month sub-study (Asia, Europe, and Latin America), the efficacy and safety of converting stable renal transplant recipients $(n=564)$ from MMF to a bioequivalent dose of EC-MPS were evaluated. ${ }^{73}$ Patients who were at least at 3 months post transplantation (ie, maintenance patients) and had a stable graft function under CsA-MMF with or without corticosteroids therapy were eligible to enter the subprotocol. After switch from MMF to EC-MPS, renal function remained stable throughout the trial (baseline calculated creatinine clearance $65.3 \pm 20.4 \mathrm{~mL} / \mathrm{min}$ and $66.9 \pm 21.4 \mathrm{~mL} / \mathrm{min}$ at month 6 ) and the rate of treatment failure was low (1.9\% of which $1.7 \%$ biopsy-proven acute rejection within 6 months of converting to study medication, no episodes of graft loss). EC-MPS was well tolerated with only mild to moderate severity adverse-events (6.5\% hematological, $23.5 \% \mathrm{GI})$, leading to dose reductions $(6.3 \%)$ or drug interruption $(3.4 \%)$. The results of this large multi-center study provided further proof of the safety and maintained efficacy of EC-MPS compared to MMF in maintenance immunosuppression for renal transplant recipients.

Another pooled analysis of three myPROMS sub-studies (US01, DE01, FR01 substudies performed in USA, Germany and France, respectively) evaluated the efficacy and tolerability of EC-MPS de novo in renal transplantation. All patients received steroids and basiliximab induction and were randomized in standard or low-dose CsA, and early (day 0) or delayed (day 6 after transplantation) CsA introduction treatment groups. ${ }^{74}$ There was $25.9 \%$ treatment failure reported at 12 months after transplantation $(22.1 \%$ biopsy-proven acute rejection, 3.1\% graft loss) and renal function was stable (median calculated creatinine clearance $62.9 \mathrm{~mL} / \mathrm{min}$ ). There was no significant difference between CsA treatment groups at month 6 and 12 in terms of biopsyproven acute rejection, graft and patient survival and graft function. High EC-MPS dosing was sustained throughout the study period ( $>90 \%$ recommended dose) and dose modifications due to EC-MPS-related adverse-events or infections were infrequent. Similarly, we have analysed a sub-group of 140 de novo kidney-transplant recipients from 11 centers in Europe, treated with EC-MPS and CsA microemulsion with or without steroids. ${ }^{75}$ The incidence of treatment failure, 
biopsy-proven acute rejection and graft loss was comparable at 6 and 12 months $(18.6 \%$ vs $22.1 \%, 15.7 \%$ vs $19.3 \%$, and $1.4 \%$ vs $2.1 \%$, at 6 and 12 months, respectively) with preserved renal function (calculated creatinine clearance $63.2 \pm 27.1 \mathrm{~mL} / \mathrm{min}$ at $12 \mathrm{months}$ ). In summary, these pooled analysis have shown that EC-MPS given in combination with CsA microemulsion, steroids and IL-2-receptor antagonist induction offers effective and well-tolerated immunosuppression in de novo renal transplantation.

\section{MPA exposure and therapeutic drug monitoring}

Based on the results of pivotal large prospective randomized trials, MMF was approved for the prevention of acute rejection in renal transplantation in combination with CsA and steroids. ${ }^{22-24}$ But unlike other maintenance immunosuppressive drugs such as CNI or mTOR inhibitors, MMF was introduced into routine clinical practice on a fixed-dose recommendation ( 2 or $3 \mathrm{~g}$ /day). As discussed, these standard-dose regimens have overall proven to be efficient in preventing early acute rejection as well as late rejection and graft failure. However, it has been shown that for an identical oral dose of MMF, there is an intra- and inter-patient variability of the pharmacokinetics of MPA. Indeed, factors such as renal function, serum albumin and bilirubine, concurrent medication (in particular CsA), altered GI absorption, and host genetics may influence MPA exposure. ${ }^{76,77}$ Thus, fixed-dose MMF therapy may lead to under- or over-immunosuppression leading to increased risk of acute rejection or drug toxicity, respectively. Clinical studies have reported a significant correlation between incidence of acute rejection and MPA plasma concentrations. ${ }^{78-80}$ Therapeutic drug monitoring of the active metabolite MPA pharmacokinetic parameters would allow optimizing the efficacy and safety of immunosuppression and a more individualized-based treatment. ${ }^{81}$

The most reliable measure of MPA exposure is the 12-hour area under the plasma concentration-time curve $\left(\mathrm{AUC}_{0-12}\right)$. The measurement however implies blood samples drawn over a 12-hour time interval and is not feasible in routine clinical practice. Predose measurement (trough levels) of MPA is an alternative to AUC, but is less accurate due to greater within-patient variability. Simplified limited sampling AUC strategies have been proposed and evaluated in renal transplant patients at various time-points after transplantation. ${ }^{80,82-84}$ These strategies have been shown to accurately estimate individual MPA AUC using a limited number of blood samples and are now being used for MPA dose-adaptation in concentration-controlled studies in clinical practice. Moreover, as the biological activity of MPA may be more relevant than its pharmacokinetic parameters in correlating with clinical outcome, an assay based on the measurement of IMP deshydrogenase activity in peripheral blood mononuclear cells has been reported. ${ }^{85}$ The benefit of TDM in renal transplantation outcome was evaluated in a 12-month prospective French trial (APOMYGRE), with 137 transplant recipients randomized to receive either concentration-controlled or fixed-dose MMF, together with basiliximab induction, CSA and steroids. ${ }^{80}$ The incidence of treatment failure (a composite of death, graft loss, acute biopsy-proven rejection, MMF discontinuation) was significantly lower in the concentration-controlled group (29.2\% vs $47.7 \%)$, which interestingly received a higher MMF dose in the first 3 months after transplantation. Compared to $\mathrm{MMF}$, equimolar doses of EC-MPS result in similar MPA exposure, but there are less data available on TDM for EC-MPS and the correlation with clinical outcome. ${ }^{86}$

Because of technical difficulties, costs and concerns about the optimal methodology to use, controversies exist whether TDM of the active metabolite MPA should be applied to all renal transplant recipients. ${ }^{87,88}$ Based on the association between clinical events and MPA exposure and the known pharmacokinetic variability, TDM has been recommended in defined clinical settings to establish adequate individualized MPA levels. ${ }^{89}$ In the first weeks after transplantation, patients with concurrent $\mathrm{CsA}$ administration were found to have MPA AUC below target levels with MMF 2 g/day. CSA, as opposed to TAC, inhibits the enterohepatic recirculation of MPA, resulting in lower MPA concentrations and potential under-exposure. Thus, TDM would be useful in the immediate post transplantation period, in high immunological risk patients, when reducing or withdrawing CSA or switching from/to another immunosuppressant such as TAC or mTOR inhibitors. It would also allow to monitor the overall degree of immunosuppression during a clinical event such as rejection or the occurrence of severe side-effects.

\section{Conclusion and perspectives}

In order to improve allograft and patient survival, the main objective of current immunosuppressive regimens is to prevent acute rejection episodes which may negatively impact on short and long-term outcomes, while limiting drug-related toxicity including CAN. Because of the good safety profile and great efficacy of MMF demonstrated in large randomized pivotal trials, most transplantation programs now routinely use MPA formulations rather than AZA in their immunosuppressive maintenance regimens. 
The use of MPA formulations, both MMF and EC-MPS, can however be limited by their side-effects (mainly GI and hematological) especially in advanced renal failure, as well as higher costs compared to AZA. In this regard, it is worth noting that the early prospective randomized trials that have lead to "standard" CsA-MMF-prednisone triple therapy had used an old formulation of CsA. A recent multi-center prospective randomized cadaveric renal transplantation study (Mycophenolate Steroids Sparing, MYSS trial), comparing the efficacy of MMF ( $2 \mathrm{~g} /$ day) to AZA (75-100 mg/day) in combination with the CsA-microemulsion formulation (Neoral) and steroids showed no clear advantage of MMF over AZA in preventing early ( $<6$ months after transplantation) as well as late ( $>6$ months after transplantation) occurring acute rejection..$^{90}$ In the extension of MYSS, the follow-up study, graft function, incidence of late rejections and adverse events, graft and patient survival were comparable among both treatment groups at 5-years follow-up. The authors concluded that in kidney transplantation, short as well as long-term efficacy profiles of MMF and AZA therapy in combination with CsA-microemulsion were similar. ${ }^{91}$ They also pointed out that MMF treatment was about 15 times more expensive.

Overall, the addition and type of induction therapy, the type and dosage of CNI versus other anti-proliferative agents such as mTOR-inhibitors in maintenance immunosuppressive regimens together with MMF may impact differently on the rate of acute allograft rejection and graft survival. Further large prospective long-term studies are needed to better determine the combination therapy that would provide optimal graft and patient outcome. In addition, treatment based on TDM would provide individualized immunosuppression and may help optimize the efficacytoxicity profile of immunosuppressive drugs and further improve clinical outcome. Cost-effectiveness should also be an issue in the choice of medication as well as the type of monitoring of standard immunosuppressive regimens for renal transplant recipients.

\section{Acknowledgments}

D Golshayan is supported by a SCORE grant $\left(\mathrm{N}^{\circ} 3232 \mathrm{BO}-\right.$ 111370/1) from the Swiss National Science Foundation, and a grant from the Faculty of Biology and Medicine of Lausanne University. B Vogt is supported by a grant from the Swiss National Science Foundation (N 3100A0-120406/1).

\section{Disclosures}

The authors disclose no conflicts of interest.

\section{References}

1. Sayegh MH, Carpenter CB. Transplantation 50 years later-progress, challenges, and promises. N Engl J Med. 2004;351(26):2761-2766.

2. Halloran PF. Immunosuppressive drugs for kidney transplantation. N Engl J Med 2004;351(26):2715-2729.

3. Meier-Kriesche HU, Li S, Gruessner RW, et al. Immunosuppression: evolution in practice and trends, 1994-2004. Am J Transplant. 2006;6:1111-1131.

4. Magee CC, Pascual M. Update in renal transplantation. Arch Intern Med. 2004;164:1373-88.

5. Burke JF Jr, Pirsch JD, Ramos EL, et al. Long-term efficacy and safety of cyclosporine in renal-transplant recipients. $N$ Engl $J$ Med. 1994;331:358-363.

6. Hariharan S, Johnson CP, Bresnahan BA, Taranto SE, McIntosh MJ, Stablein D. Improved graft survival after renal transplantation in the United States, 1988 to 1996. N Engl J Med. 2000;342:605-612.

7. Pascual M, Theruvath T, Kawai T, Tolkoff-Rubin N, Cosimi AB. Strategies to improve long-term outcomes after renal transplantation. N Engl J Med. 2002;346(8):580-590.

8. Meier-Kriesche HU, Schold JD, Srinivas TR, Kaplan B. Lack of improvement in renal allograft survival despite a marked decrease in acute rejection rates over the most recent era. Am J Transplant. 2004;4:378-383.

9. Hernandez-Fuentes MP, Lechler RI. Chronic graft loss. Immunological and non-immunological factors. Contrib Nephrol. 2005;146:54-64.

10. Nankivell BJ, Borrows RJ, Fung CL, O'Connell PJ, Allen RD, Chapman JR. The natural history of chronic allograft nephropathy. N Engl J Med. 2003;349:2326-2333.

11. Allison AC, Eugui EM. Mycophenolate mofetil, a rationally designed immunosuppressive drug. Clin Transplant. 1993;7:96-112.

12. Florey HW, Gilliver K, Jennings MA. Mycophenolic acid: an antibiotic from Penicillium brevicompactum. Lancet. 1946;1:46-49.

13. Carter SB, Franklin TJ, Jones DF, et al.Mycophenolic acid: an anticancer compound with unusual properties. Nature. 1969;223:848.

14. Lipsky J. Mycophenolate mofetil. Lancet. 1996;348:1357-1359.

15. Sintchak MD, Fleming MA, Futer O, et al. Structure and mechanism of inosine monophosphate dehydrogenase in complex with the immunosuppressant mycophenolic acid. Cell. 1996;85:921-930.

16. Morris R, Hoyt E, Murphy M, Eugui E, Allison A. Mycophenolic acid morpholinoethyl ester (RS-61443) is a new immunosuppressant that prevents and halts heart allograft rejection by selective inhibition of T- and B-cell purine synthesis. Transplant Proc. 1990;22:1659-1662.

17. Allison AC, Kowalski WJ, Muller CJ, Waters RV, Eugui EM. Mycophenolic acid and Brequinar, inhibitors of purine and pyrimidine synthesis, block the glycosylation of adhesion molecules. Transplant Proc. 1993;25(S2):67-70.

18. Bullingham RE, Monroe S, Nicholls A, Hale M. Pharmacokinetics and bioavailability of mycophenolate mofetil in healthy subjects after single-dose oral and intravenous administration. J Clin Pharmacol. 1996;36:315-324.

19. Arns W, Breuer S, Choudhury S, et al. Enteric-coated mycophenolate sodium delivers bioequivalent MPA exposure compared with mycophenolate mofetil. Clin Transplant. 2005;19:199-206.

20. Tedesco-Silva H, Bastien MC, Choi L, et al. Mycophenolic acid metabolite profile in renal transplant patients receiving enteric-coated mycophenolate sodium or mycophenolate mofetil. Transplant Proc. 2005;37(2):852-855.

21. Johnston A, He X, Holt DW. Bioequivalence of enteric-coated mycophenolate sodium and mycophenolate mofetil: a meta-analysis of three studies in stable renal transplant recipients. Transplantation. 2006;82(11):1413-1418.

22. European Mycophenolate Mofetil Study Group. Placebo-controlled study of mycophenolate mofetil combined with cyclosporine and corticosteroids for prevention of acute rejection. Lancet. 1995;345: 1321-1325. 
23. Tricontinental Mycophenolate Mofetil Renal Transplantation Study Group. A blinded, randomized clinical trial of mycophenolate mofetil for the prevention of acute rejection in cadaveric renal transplantation. Transplantation. 1996;61:1029-1037.

24. US Renal Transplant Mycophenolate Mofetil Study Group, Sollinger HW. Mycophenolate mofetil for the prevention of acute rejection in primary cadaveric renal allograft recipients. Transplantation. 1995;60:225-232.

25. The International Mycophenolate Mofetil Renal Transplant Study Groups, Halloran PF, Mathew T, Tomlanovich S, Groth C, Hooftman L, Barker C. Mycophenolate mofetil in renal allograft recipients: a polled efficacy analysis of three randomized, double-blind, clinical studies in prevention of rejection. Transplantation. 1997;63:39-47.

26. Tricontinental Mycophenolate Mofetil Renal Transplantation Study Group, Mathew TH. A blinded, long-term, randomized multi-center study of mycophenolate mofetil in cadaveric renal transplantation. Results at three years. Transplantation. 1998;65:1450-1454.

27. European Mycophenolate Mofetil Cooperative Study Group. Mycophenolate mofetil in renal transplantation: 3-year results from the placebo-controlled trial. Transplantation. 1999;68:391-396.

28. Cecka JM, Cho YW, Terasaki PI. Analyses of the UNOS scientific renal transplant registry at three years - early events affecting transplant success. Transplantation. 1992;53:59-64.

29. Sadek S, Medina J, Arias M, Sennesael J, Squifflet JP, Vogt B; Neo Int-05 Study Group. Short-term combination of mycophenolate mofetil with cyclosporine as a therapeutic option for renal transplant recipients: a prospective, muticenter, randomized study. Transplantation. 2002; 74(4):511-517.

30. Mycophenolate Mofetil Renal Refractory Rejection Study Group. Mycophenolate mofetil for the treatment of refractory, acute, cellular renal transplant rejection. Transplantation. 1996;61:722-728.

31. Mycophenolate Mofetil Acute Renal Rejection Study Group. Mycophenolate mofetil for the treatment of a first acute renal allograft rejection. Transplantation. 1998;65:235-241.

32. Mycophenolate Mofetil Acute Renal Rejection Study Group. Mycophenolate mofetil for the treatment of a first acute renal allograft rejection: three-year follow-up. Transplantation. 2001;71:1091-1097.

33. Meier-Kriesche HU, Steffen BJ, Hochberg AM, et al. Long-term use of mycophenolate mofetil is associated with a reduction in the incidence and risk of late rejection. Am J Transplant. 2003;3(1):68-73.

34. Zucker K, Tsaroucha A, Olson L, Esquenazi V, Tzakis A, Millers J. Evidence that tacrolimus augments the bioavailability of mycophenolate mofetil through the inhibition of mycophenolic acid glucuronidation. Ther Drug Monitor. 1999;21:35-43.

35. Shapiro R, Jordan ML, Scantlebury VP, et al. A prospective, randomized trial of tacrolimus/prednisone versus tacrolimus/prednisone/ mycophenolate mofetil in renal transplant recipients. Transplantation. 1999;67:411-415.

36. Miller J, Mendez R, Pirsch JD, Jensik SC. Safety and efficacy of tacrolimus in combination with mycophenolate mofetil (MMF) in cadaveric renal transplant recipients. FK506/MMF Dose-Ranging Kidney Transplant Study Group. Transplantation. 2000;69:875-879.

37. Johnson C, Ahsan N, Gonwa T, et al. Randomized trial of tacrolimus (Prograf) in combination with azathioprine or mycophenolate mofetil versus cyclosporine (Neoral) with mycophenolate mofetil after cadaveric kidney transplantation. Transplantation. 2000;69:834-841.

38. Ahsan N, Johnson C, Gonwa T, et al. Randomized trial of tacrolimus plus mycophenolate mofetil or azathioprine versus cyclosporine oral solution (modified) plus mycophenolate mofetil after cadaveric kidney transplantation: results at 2 years. Transplantation. 2001;72(2):245-250.

39. Gonwa T, Johnson C, Ahsan N, et al. Randomized trial of tacrolimus + mycophenolate mofetil or azathioprine versus cyclosporine + mycophenolate mofetil after cadaveric kidney transplantation: results at three years. Transplantation. 2003;75:2048-2053.

40. Ekberg H, Tedesco-Silva H, Demirbas A, et al; ELITE-Symphony Study. Reduced exposure to calcineurin inhibitors in renal transplantation. N Engl J Med. 2007;357(25):2562-2575.
41. Bunchman T, Navarro M, Broyer M, et al. The use of mycophenolate mofetil suspension in pediatric renal allograft recipients. Pediatr Nephrol. 2001;16:978-984.

42. Neylan JF. Immunosuppressive therapy in high-risk transplant patients: dose-dependent efficacy of mycophenolate mofetil in African-American renal allograft recipients. US Renal Transplant Mycophenolate Mofetil Study Group. Transplantation. 1997;64(9):1277-1282.

43. Cianco G, Burke GW, Suzart K, et al. The use of daclizumab, tacrolimus and mycophenolate mofetil in african-american and Hispanic first renal transplant recipients. Am J Transplant. 2003;3(8): 1010-1016.

44. Golshayan D, Pascual M. Minimization of calcineurin inhibitors to improve long-term outcomes in kidney transplantation. Transplant Immunol. 2008; Sep 4. [Epub ahead of print].

45. Meier-Kriesche HU, Steffen BJ, Hochberg AM, et al. Mycophenolate mofetil versus azathioprine therapy is associated with a significant protection against long-term renal allograft function deterioration. Transplantation 2003;75:1341-1346.

46. Ojo AO, Meier-Kriesche HU, Hanson JA, et al. Mycophenolate mofetil reduces late renal allograft loss independent of acute rejection. Transplantation. 2000;69:2405-2409.

47. Smak Gregoor PJ, de Sevaux RG, Ligtenberg G, et al. Withdrawal of cyclosporine or prednisone six months after kidney transplantation in patients on triple drug therapy: a randomized, prospective, multicenter study. J Am Soc Nephrol. 2002;13:1365-1373.

48. Abramowicz D, Del Carmen RM, Vitko S, et al; Cyclosporine Withdrawal Study Group. Cyclosporine withdrawal from a mycophenolate mofetil-containing immunosuppressive regimen: results of a five-year, prospective, randomized study. J Am Soc Nephrol. 2005;16:2234-2240.

49. Pascual M, Curtis J, Delmonico FL, et al. A prospective, randomized clinical trial of cyclosporine reduction in stable patients greater than 12 months after renal transplantation. Transplantation. 2003;76(8):1240-1241.

50. Ekberg H, Grinyo J, Nashan B, et al. Cyclosporine sparing with mycophenolate mofetil, daclizumab and corticosteroids in renal allograft recipients: the CAESAR Study. Am J Transplant. 2007:7:560-570.

51. Grinyo JM, Gil-Vernet S, Cruzado JM, et al. Calcineurin inhibitor-free immunosuppression based on antithymocyte globulin and mycophenolate mofetil in cadaveric kidney transplantation: results after 5 years. Transpl Int. 2003;16:820-827.

52. Pearson TC, Mulgaonkar S, Patel A, et al. Efficacy and safety of mycophenolate mofetil/sirolimus maintenance therapy after calcineurin inhibitor withdrawal in renal transplant recipients: final results of the Spare-the-Nephron (STN) Trial. Am J Transplant. 2008;8(S2):213.

53. Vincenti F, Larsen C, Durrbach A, et al; Belatacept Study Group. Costimulation blockade with belatacept in renal transplantation. $N$ Engl J Med. 2005;353(8):770-781.

54. Hueso M, Bover J, Seron D, et al. Low-dose cyclosporine and mycophenolate mofetil in renal allograft recipients with suboptimal renal function. Transplantation 1998;66(12):1727-1731.

55. Pascual M, Williams WW, Cosimi AB, Delmonico FL, Farrell ML, Tolkoff-Rubin N. Chronic renal allograft dysfunction: a role for mycophenolate mofetil? Transplantation. 2000;69:1749-1750.

56. Afzali B, Shah S, Chowdhury P, O'Sullivan H, Taylor J, Goldsmith D. Low-dose mycophenolate mofetil is an effective and safe treatment to permit phased reduction in calcineurin inhibitors in chronic allograft nephropathy. Transplantation. 2005;79:304-309.

57. Ducloux D, Motte G, Billerey C, et al. Cyclosporine withdrawal with concomitant conversion from azathioprine to mycophenolate mofetil in renal transplant recipients with chronic allograft nephropathy: a 2-year follow-up. Transpl Int. 2002;15:387-392.

58. Weir MR, Ward MT, Blahut SA, et al. Long-term impact of discontinued or reduced calcineurin inhibitor in patients with chronic allograft nephropathy. Kidney Int. 2001;59(4):1567-1573. 
59. Dudley C, Pohanka E, Riad H, et al; Mycophenolate Mofetil Creeping Creatinine Study group. Mycophenolate mofetil substitution for cyclosporine A in renal transplant recipients with chronic progressive allograft dysfunction: the "creeping creatinine" study. Transplantation. 2005;79:466-475.

60. Suwelack B, Gerhardt U, Hohage H. Withdrawal of cyclosporine or tacrolimus after addition of mycophenolate mofetil in patients with chronic allograft nephropathy. Am J Transplant. 2004;4:655-662.

61. Frimat L, Cassuto-Viguier E, Charpentier B, et al. Impact of cyclosporine reduction with MMF: a randomized trial in chronic allograft dysfunction. The 'Reference' Study. Am J Transplant. 2006;6:2725-2734.

62. Groth CG, Backman L, Morales JM, et al. Sirolimus (rapamycin)-based therapy in human renal transplantation: similar efficacy and different toxicity compared with cyclosporine. Sirolimus European Renal Transplant Study Group. Transplantation. 1999;67:1036-1042.

63. Kreis H, Cisterne JM, Land W, et al. Sirolimus in association with mycophenolate mofetil induction for the prevention of acute graft rejection in renal allograft recipients. Transplantation. 2000;69(7):1252-1260.

64. Flechner SM, Kurian SM, Solez K, et al. De novo kidney transplantation without use of calcineurin inhibitors preserves renal structure and function at two years. Am J Transplant. 2004;4:1776-1785.

65. Larson TS, Dean PG, Stegall MD, et al. Complete avoidance of calcineurin inhibitors in renal transplantation: a randomized trial comparing sirolimus and tacrolimus. Am J Transplant. 2006;6:514-522.

66. Epinette WW, Parker CM, Jones EL, Griest MC. Mycophenolic acid for psoriasis. A review of pharmacology, long-term efficacy and safety. J Am Acad Dermatol. 1987;17:962-971.

67. Behrend M. Adverse gastrointestinal effects of mycophenolate mofetil: etiology, incidence and management. Drug Saf. 2001;24:645-663.

68. Chan L, Mulgaonkar S, Walker R, Arns W, Ambuhl P, Schiavelli R. Patient-reported gastrointestinal symptom burden and health-related quality of life following conversion from mycophenolate mofetil to enteric-coated mycophenolate sodium. Transplantation. 2006;81: 1290-1297.

69. Bolin P, Tanriover B, Zibari GB, et al. Improvement in 3-month patient-reported gastrointestinal symptoms after conversion from mycophenolate mofetil to enteric-coated mycophenolate sodium in renal transplant patients. Transplantation. 2007;84(11):1443-1451.

70. Salvadori M, Holzer H, de Mattos A, et al; the ERL B301 Study Grooups. Enteric-coated mycophenolate sodium is therapeutically equivalent to mycophenolate mofetil in de novo renal transplant patients. Am J Transplant. 2004;4:231-236.

71. Salvadori M, Holzer H, Civati G, et al. ERL B301 Study Group. Long-term administration of enteric-coated mycophenolate sodium (EC-MPS; myfortic) is safe in kidney transplant patients. Clin Nephrol. 2006;66(2):112-119.

72. Budde K, Knoll G, Curtis J, et al; ERL B302 Study Group. Long-term safety and efficacy after conversion of maintenance renal transplant recipients from mycophenolate mofetil (MMF) to entericcoated mycophenolate sodium (EC-MPA, myfortic). Clin Nephrol. 2006;66(2):103-111.

73. Pietruck F, Abbud-Filho M, Vathsala A, Massari PU, Po-Huang L, Nashan B. Conversion from mycophenolate mofetil to enteric-coated mycophenolate sodium in stable maintenance renal transplant patients: pooled results from three international, multicenter studies. Transplant Proc. 2007;39(1):103-108.

74. Legendre C, Cohen D, Zeier M, Rostaing L, Budde K. Efficacy and safety of enteric-coated mycophenolate sodium in de novo renal transplant recipients: pooled data from three 12-month multicenter, openlabel, prospective studies. Transplant Proc. 2007;39(5):1386-1391.
75. Vogt B, Antoniadis A, Klinger M, Vitko S. Efficacy and safety of enteric-coated mycophenolate sodium (myfortic) in de novo renal transplant recipients: results of a 12-month multicenter, open-label, prospective study. Transplant Proc. 2006;38:1301-1306.

76. Nowak I, Shaw LM. Mycophenolic acid binding to human serum albumin: characterization and relation to pharmacodynamics. Clin Chem. 1995;41(7):1011-1017.

77. Kuypers DR. Influence of interactions between immunosuppressive drugs on therapeutic monitoring. Ann Transplant. 2008;13(3):11-18.

78. Van Gelder T, Hilbrands LB, Vanrenterghem Y, et al. A randomized double-blind, multicenter plasma concentration controlled study of the safety and efficacy of oral mycophenolate mofetil for the prevention of acute rejection after kidney transplantation. Transplantation. 1999;68(2):261-266.

79. Kiberd BA, Lawen J, Fraser AD, Keough-Ryan T, Belitsky P. Early adequate mycophenolic acid exposure is associated with less rejection in kidney transplantation. Am J Transplant. 2004;4(7):1079-1083.

80. Le Meur Y, Büchler M, Thierry A, et al. Individualized mycophenolate mofetil dosing based on drug exposure significantly improves patient outcomes after renal transplantation. Am J Transplant. 2007;7(11):2496-2503.

81. van Gelder T, Shaw LM. The rationale for and limitations of therapeutic drug monitoring for mycophenolate mofetil in transplantation. Transplantation 2005;80(2 Suppl):S244-S53.

82. Willis C, Taylor PJ, Salm P, Tett SE, Pillans PI. Evaluation of limited sampling strategies for estimation of 12-hour mycophenolic acid area under the plasma concentration-time curve in adult renal transplant patients. Ther Drug Monit. 2000;22(5):549-554.

83. Prémaud A, Debord J, Rousseau A, et al. A double absorption-phase model adequately describes mycophenolic acid plasma profiles in de novo renal transplant recipients given oral mycophenolate mofetil. Clin Pharmacokinet. 2005;44(8):837-847.

84. Prémaud A, Le Meur Y, Debord J, et al. Maximum a posteriori bayesian estimation of mycophenolic acid pharmacokinetics in renal transplant recipients at different postgrafting periods. Ther Drug Monit. 2005;27(3):354-361.

85. Glander P, Braun KP, Hambach P, et al. Non-radioactive determination of inosine $5^{\prime}$-monophosphate dehydro-genase (IMPDH) in peripheral mononuclear cells. Clin Biochem. 2001;34(7):543-549.

86. Budde K, Bauer S, Hambach P, et al. Pharmacokinetic and pharmacodynamic comparison of enteric-coated mycophenolate sodium and mycophenolate mofetil in maintenance renal transplant patients. Am J Transplant. 2007;7(4):888-898.

87. Shaw LM, Holt DW, Oellerich M, Meiser B, van Gelder T. Current issues in therapeutic drug monitoring of mycophenolic acid: report of a roundtable discussion. Ther Drug Monit. 2001;23(4):305-315.

88. West-Thielke P, Kaplan B. Therapeutic monitoring of mycophenolic acid: is there clinical utility? Am J Transplant. 2007;7(11): 2441-2442.

89. Shaw LM, Korecka M, Venkataramanan R, Goldberg L, Bloom R, Brayman KL. Mycophenolic acid pharmacodynamics and pharmacokinetics provide a basis for rational monitoring strategies. Am J Transplant. 2003;3(5):534-542.

90. Remuzzi G, Lesti M, Gotti E, et al. Mycophenolate mofetil versus azathioprine for prevention of acute rejection in renal transplantation (MYSS): a randomised trial. Lancet. 2004;364:503-512.

91. Remuzzi G, Cravedi P, Costantini M, et al. Mycophenolate mofetil versus azathioprine for prevention of chronic allograft dysfunction in renal transplantation: the MYSS follow-up randomized, controlled clinical trial. J Am Soc Nephrol. 2007;18(6):1973-1985. 
